\title{
ON THE STRUCTURE OF A TRIANGLE-FREE INFINITE-CHROMATIC GRAPH OF GYARFAS
}

\author{
LARRY EGGAN \\ Mathematical Reviews \\ Ann Arbor, MI 48109 \\ I11inois State University \\ Norma1, IL 61761 \\ FRANK HARARY \\ Churchill College \\ Cambridge CV3 oDS, England \\ University of Michigan \\ Ann Arbor, MI 48109, USA
}

(Received June 22, 1981 and in revised form September 13, 1982)

ABSTRACT. Gyárfás has recently constructed an elegant new example of a trianglefree infinite graph $G$ with infinite chromatic number. We analyze its structure by studying the properties of a nested family of subgraphs $G_{n}$ whose union is $G$.

KEY WORDS AND PHRASES: Triangle-free, infinite-chromatic

\section{MATHEMATICAL SUBJECT CLASSIFICATION CODE: $05 \mathrm{C} 15$}

1. INTRODUCTION.

Gyárfás [1] described a new example of a triangle-free infinite-chromatic graph $G$ as follows: the vertices of $G$ form an $\infty \times \infty$ matrix, i.e., $V=\left\{v_{i, j} ; i, j=\right.$ $1,2, \ldots\}$, and the vertex $v_{i, j}$ is adjacent to every vertex of the $(i+j)$-th column, i.e., the set $E$ of edges of $G$ is given by $E=\left\{v_{i, j} v_{k, i+j} ; i, j, k=1,2, \ldots\right\}$. It is easy to see that $G$ is triangle-free for if $u, v, w$, were vertices of a triangle with u having the smallest column index, then the fact that uv and uw are edges would mean 
$v$ and $w$ are adjacent vertices in the same column, which is impossible. That $G$ requires infinitely many colors follows from Theorem 2 below, although it also follows directly from the fact that, for $j>i$, the $i-t$ th column contains a vertex adjacent to all vertices of the $j$-th column.

In what follows we will accomplish two things. We first describe an augmenting sequence of finite graphs, which has $G$ as its limit, and determine the structure of these graphs. This gives a deeper insight into the actual structure of G. Unless otherwise specified, we follow the graph theoretic notation and terminology of Harary [2].

\section{AN ANALYSIS OF G.}

In this section we will define a sequence $G_{n}$ of graphs converging to $G$. We will give some results on the structure of each $G_{n}$, and an alternative way of constructing $G_{n}$ which gives a different perspective on its structure. Finally, we will note that the desired properties of $G_{n}$ can be demonstrated by considering subgraphs $H_{n}$ (which are roughly half of $G_{n}$ ).

DEFINITION. For any positive integer $n$, let $G_{n}$ be the subgraph of $G$ obtained by removing all vertices $v_{i, j}$ with $i$ and $j$ greater than $n$, i.e., $G_{n}$ is the induced subgraph $\left\langle\left\{v_{i, j} ; 1 \leq i, j \leq n\right\}\right\rangle$ of $G$.

First we prove a theorem on the degrees of the vertices of $G_{n}$.

THEOREM 1. For $0 \leq k \leq 2 n-2$, the number of vertices of $G_{n}$ of degree $k$ is $n-|n-k-1|$, while there are no vertices of degree greater than $2 n-2$.

PROOF. Consider $G_{n}$ as an $n \times n$ matrix. Then it is easy to see that

$$
\operatorname{deg}\left(v_{i, j}\right)= \begin{cases}n+j-1, & \text { if } 1 \leq i \leq n-j \\ j-1, & \text { if } n-j+1 \leq i \leq n .\end{cases}
$$

Thus by setting $k=n+j-1$ or $k=j-1$, we see there are either $2 n-k-1$ or $k+1$ vertices, respectively, of order $k$, as claimed.

In the next theorem we give the chromatic number $x\left(G_{n}\right)$ of each $G_{n}$. 
THEOREM 2. For $k \geq 1, G_{n}$ is $k$-colorable if $n<2^{k}$, while $G_{2^{k}}$ has chromatic number $k+1$, that is, $\chi\left(G_{n}\right)=1+\left[\log _{2} n\right]$.

PROOF. Since $G_{n-1}$ is a subgraph of $G_{n}$, it suffices to show that $G_{2} k_{-1}$ is $k$ colorable whereas $G_{2} k$ is not. To show the latter, suppose on the contrary that $G_{2} k$ is colored in $k$ colors, and let $N_{j}$ denote the set of colors used on the vertices in the $j$-th column of $G_{2 k}$. Now for $i<j, v_{j-i, i}$ is adjacent to every vertex in column $j$ so $N_{i} \nsubseteq N_{j}$. The sets $N_{j}$ thus form a collection of $2^{k}$ distinct nonempty subsets of a k-element set, which is impossible.

To show that $G_{2} k_{-1}$ is $k$-colorable, let $C$ be a set of $k$ colors and let $N_{j}$, $j=1,2, \ldots, 2^{k}-1$, be an enumeration of the nonempty subsets of $C$ which is nonincreasing in order of size. For example, such an enumeration when $\mathrm{k}=3$ and $\mathrm{C}=$ $\left\{c_{1}, c_{2}, c_{3}\right\}$ is: $\left\{c_{1}, c_{2}, c_{3}\right\},\left\{c_{1}, c_{2}\right\},\left\{c_{1}, c_{3}\right\},\left\{c_{2}, c_{3}\right\},\left\{c_{1}\right\},\left\{c_{2}\right\}$ $\left\{c_{3}\right\}$. This enumeration provides that if $j>i$, then there is a color in $N_{i}$ which is not in $\mathrm{N}_{j}$. Therefore, color the vertex $\mathrm{v}_{\mathrm{r}, i}$ with a color in $\mathrm{N}_{i}$ which is not in $\mathrm{N}_{\mathrm{r}+\mathrm{i}}$; if $\mathrm{r}+\mathrm{i}>2^{\mathrm{k}}-1$, then use any color in $\mathrm{N}_{i}$. This clearly yields a kcoloring of $\mathrm{G}_{2} \mathrm{k}_{-1}$.

To conclude this section, we describe an alternative way to construct $G_{n}$ which we feel gives some insight into its structure and chromatic number. In accordance with established terminology, we will say that a point covers a set $S$ of points if it is adjacent to every point of $S$. A set $T$ of points smothers $S$ if exactly one point in $\mathrm{T}$ covers $\mathrm{S}$, and $\mathrm{T}$ smothers a finite sequence $\mathrm{S}_{1}, \mathrm{~S}_{2}, \ldots, \mathrm{S}_{k}$ of sets of points if there are distinct points $t_{1}, t_{2}, \ldots, t_{k}$ in $T$ such that $t_{j}$ covers $s_{j}$ for $j=$ $1,2, \ldots, \mathrm{k}$

We now describe how to construct $G_{n}$ using this idea and the join operation + , where $\mathrm{H}+\mathrm{H}^{\prime}$ is the graph obtained from the union of $\mathrm{H}$ and $\mathrm{H}^{\prime}$ by joining every point of $\mathrm{H}$ to every point of $\mathrm{H}^{\prime}$; see [2, p. 21]. We describe how to build $\mathrm{G}_{\mathrm{n}}$ in three stages. Here the notation $\mathrm{H}+\mathrm{H}^{\prime}+\mathrm{H}^{\prime \prime}$ stands for the union of two joins $\mathrm{H}^{+} \mathrm{H}^{\prime}$ and $\mathrm{H}^{\prime}+\mathrm{H}^{\prime \prime}$, and similarly for more summands each of which will be a complete graph $\mathrm{K}_{\mathrm{n}}$ or its complement, the totally disconnected graph $\overline{\mathrm{K}}_{\mathrm{n}}$.

STAGE 1: Build $\overline{\mathrm{K}}_{2}+\mathrm{K}_{1}+\overline{\mathrm{K}}_{\mathrm{n}-2}$. (Label the vertex $\mathrm{K}_{1}$ by $\mathrm{r}_{\cdot}$ ) 
STAGE 2: Replace the $j$-th point in $\overline{\mathrm{k}}_{n-2}$, numbering from bottom to top, by $s_{j}=k_{1}+\bar{k}_{n}+k_{1}$, for $j=1,2, \ldots, n-2$. (Label the left $k_{1}$ by $a_{j}$ and the right $K_{1}$ by $b_{j}$ )

STAGE 3: Replace the $\bar{K}_{n}$ in $S_{j}$ by the set of $n$ points $T^{j}$ where the adjacency in $S_{j}$ is preserved, but $T_{n}^{j}$ smothers $T_{n}^{1}, T_{n}^{2}, \ldots, T_{n}^{j-1}$, for $j=1,2, \ldots, n-2$. (Suppose that $t_{j k}$ in $T_{n}^{j}$ covers $T_{n}^{j-k}$ for $k=1,2, \ldots, j-1$.)

Figure 1 shows how the construction progresses when $n=5$. This resulting graph, when a single isolated point (corresponding to $v_{n, 1}$ ) is added, is isomorphic to $G_{n}$. We will not formally prove this, though it is easy to see that an isomorphism is obtained by mapping $r$ to $v_{1,1}, b_{j}$ to $v_{n-j, 1}, a_{j}$ to $v_{n-1-j, 2}$ (and the two vertices of degree 1 to $\left.v_{i, 2}, i=n-1, n\right)$, and $T_{n}^{j}$ to the vertices in column $n+1-j$ with $t_{j, k}$ mapping. to $v_{k, n+1-j}$. It is also easy to see that another minimal coloring (besides the one given in the proof of Theorem 2) is obtained by only using colors $c_{1}, c_{2}, \ldots, c_{i}$ to $\operatorname{color} T_{n}^{j}$ for $i \leq j<2^{i}$ and $i=1,2, \ldots, k$.

Stage 1

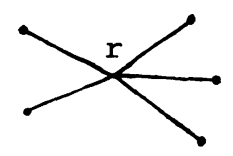

Stage 2

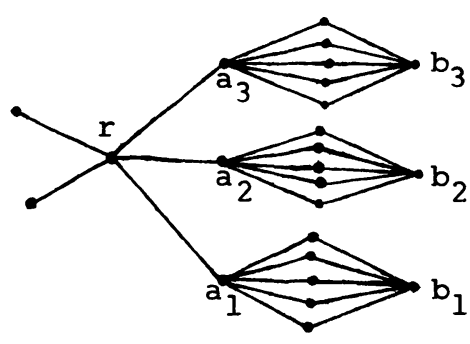

$$
\mathrm{n}=5
$$

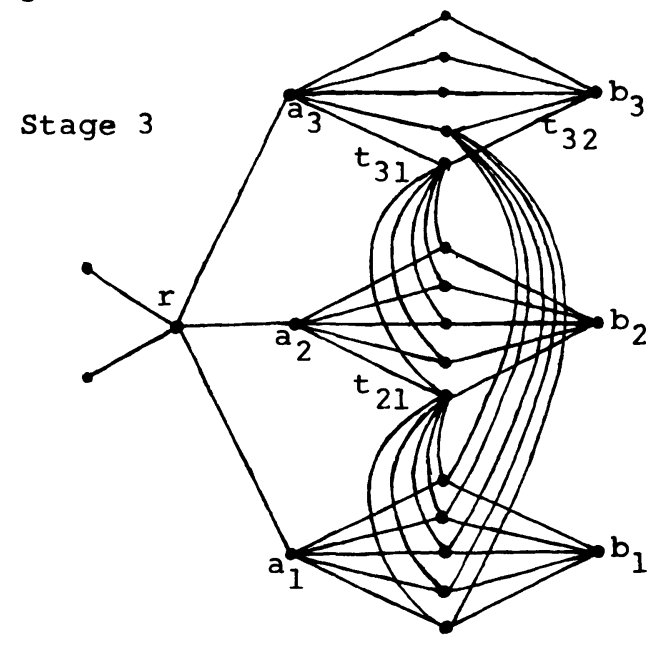

Figure 1.

Finally, let $H_{n}$ be the subgraph of $G_{n}$ with the $\frac{1}{2}\left(n^{2}-3 n+6\right)$ vertices

$$
\left\{v_{1,1}, v_{1, n}\right\} \bigcup\left\{v_{i, j} ; j=2, \ldots, n-1, i=1, \ldots, n-j\right\}
$$


It is clear that $H_{n}$ is triangle-free and for $n=2^{k}$ applying the argument in the proof of Theorem 2 to columns 2 through $n$ shows that $H_{n}$ is not $k$ colorable. Although $\mathrm{H}_{\mathrm{n}}$ is simpler then $\mathrm{G}_{\mathrm{n}}$ while still retaining the cascading appearance illustrated by Figure $1, H_{n}$ is still not critical.

We wish to thank the referee who is responsible for an improvement in our exposition and for the above proof of Theorem 2 .

\section{REFERENCES}

1. Gyárfás, A. Still another triangle-free infinite-chromatic graph, Discrete Math. $30(1980), 185$.

2. Harary, F. Graph Theory, Addison-Wesley, Reading, 1969. 


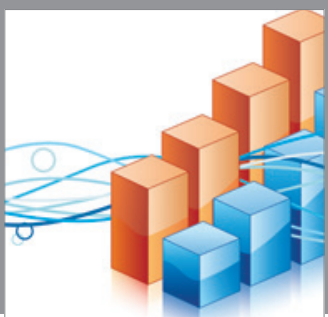

Advances in

Operations Research

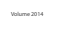

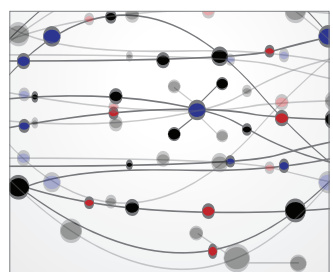

\section{The Scientific} World Journal
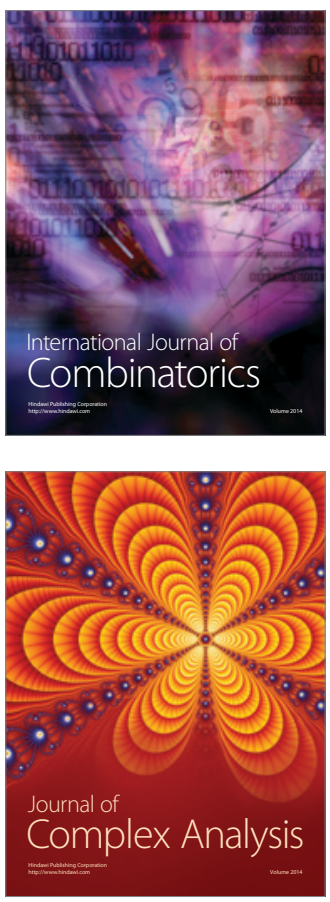

International Journal of

Mathematics and

Mathematical

Sciences
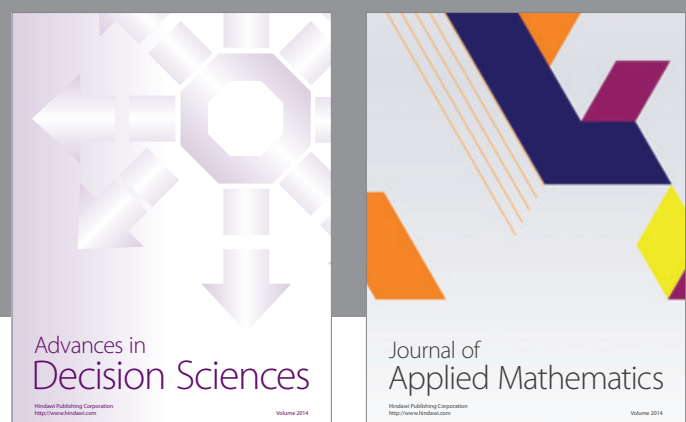

Journal of

Applied Mathematics
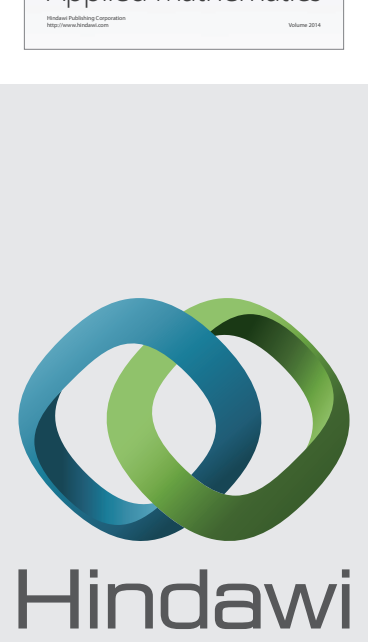

Submit your manuscripts at http://www.hindawi.com
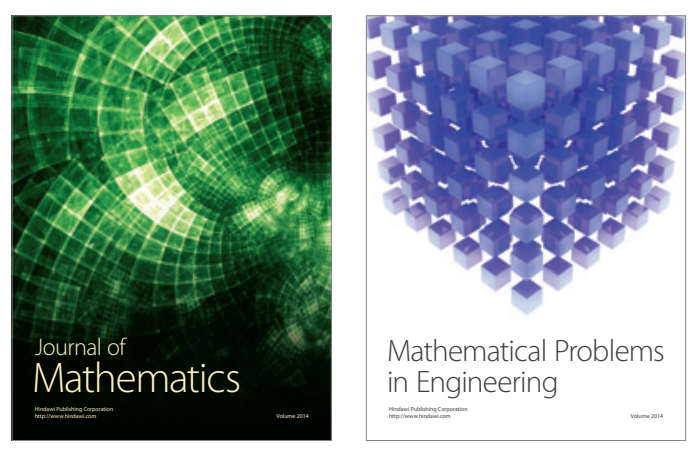

Mathematical Problems in Engineering
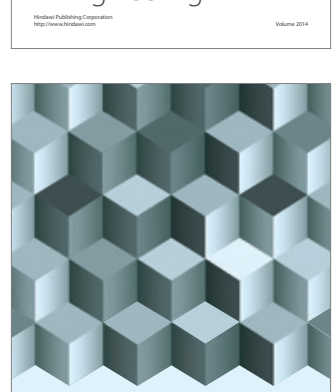

Journal of

Function Spaces
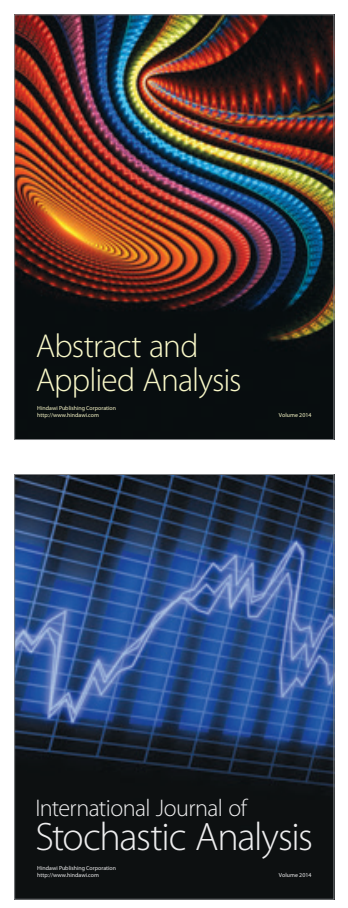

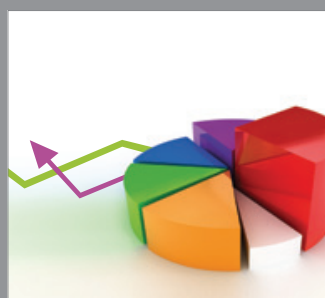

ournal of

Probability and Statistics

Promensencen
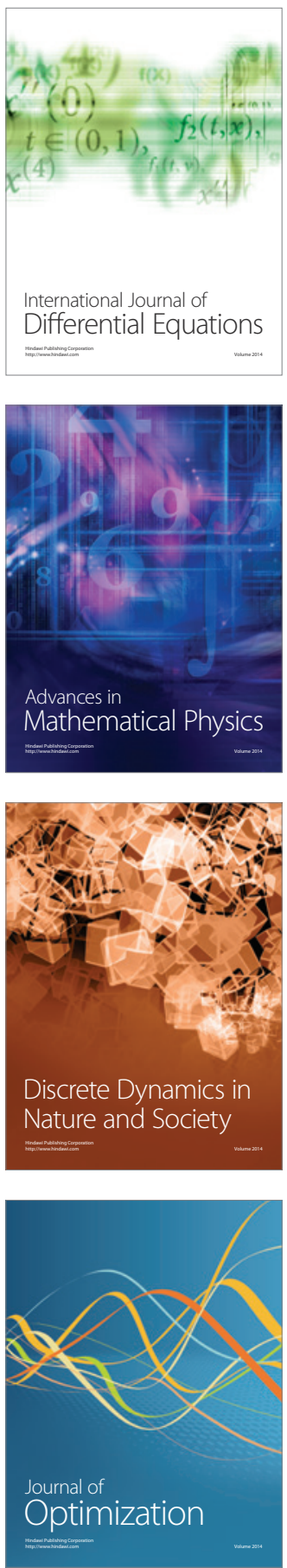